\title{
SVM classification of brain images from MRI scans using morphological transformation and GLCM texture features
}

\author{
R. Usha* and K. Perumal \\ Department of Computer Applications, \\ School of Information Technology, \\ Madurai Kamaraj University, \\ Madurai, Tamilnadu, India \\ Email: usha.resch@gmail.com \\ Email: perumalmkucs@gmail.com \\ *Corresponding author
}

\begin{abstract}
This paper introduces a novel HTT-based GLCM texture feature extraction procedure for an automatic magnetic resonance images (MRI) brain image classification. The method has three phases: 1) hierarchical transformation technique (HTT); 2) texture feature extraction; 3) classification. The new proposed HTT method incorporates optimum disk-shaped mask selection, top-hat and bottom-hat morphological operations, and some mathematical operation for both image pre-processing and enhancement. The gray level co-occurrence matrix is computed to extract statistical texture features such as contrast, correlation, energy, entropy, and homogeneity from an image. And these extracted images features of co-occurrence matrix can very well be fed into support vector machine (SVM) for further MRI brain normal and abnormal image classification. The alternate approach of the HTT-based GLCM also compared with conventional GLCM texture feature extraction method.
\end{abstract}

Keywords: magnetic resonance images; MRI; classification; texture feature extraction; grey level co-occurrence matrix; support vector machine; SVM; top hat transform; bottom hat transform.

Reference to this paper should be made as follows: Usha, R. and Perumal, K. (2019) 'SVM classification of brain images from MRI scans using morphological transformation and GLCM texture features', Int. J. Computational Systems Engineering, Vol. 5, No. 1, pp.18-23.

Biographical notes: R. Usha is pursuing PhD Research Scholar in the Department of Computer Applications, School of Information Technology, and Madurai Kamaraj University, India. She has contributed papers in national and international conferences, international journals more than six papers. Her research work is focused on medical image processing.

K. Perumal working as an Associate professor in Madurai Kamaraj University, Madurai. He has contributed more than 30 papers in international journals and conferences. He has guiding six scholars. His interest includes data mining, image processing and medical images.

\section{Introduction}

A brain tumour is an abnormal and uncontrolled growth of cells in the brain. Magnetic resonance images (MRI) has very effective feature for the detection of the brain tumour and identification due to the following reasons of the high contrast of soft tissues and the high spatial resolution compared with other medical imaging techniques such as $\mathrm{CT}, \mathrm{PET}$. And also MRI does not give harmful radiation to a human body and is a non-invasive method. Although MRI provides effective information about brain image, it cannot able to classify cancer and non-cancerous brain images. Hence the application of invasive methods such as spinal tap method and biopsy those are very painful and time-consuming process (Bandyopadhyay, 2011).

Image enhancement is one of the important processes in digital image processing to generate better-enhanced image rather than an original image for further automatic image diagnosis. The mathematical morphological technique is helpful to process and analyse the images (Maragos, 1996; Oritz and Torres, 2004; Jing et al., 2004; Peters, 1995) and it is an alternative method to shape concept. In this paper, a new morphological-based hierarchical transformation technique (HTT) technique has been used for an image enhancement. 
Features are representing images in another alternate way by measuring certain image attributes that can vary from one image to another image (D'cruz and Sudheesh, 2015). At present many features are used to analyse the medical imaging such as intensity-based features, texture features, and shape features. Among this texture image analysis can easily differentiate abnormal tissues from normal tissues. Analysis of image texture act as an important role in early diagnosis and it is used to replace the biopsy methods. The evaluations of pixel inter-relationships, in the form of texture analyses, are classified into structural, model-based, statistical and transform approaches (Materka and Strzelecki, 1998). Particularly, the statistical-based texture representations give higher discrimination indexes than other approaches such as structural and transform (Castellano et al., 2004). Texture features are helpful to show the textures characteristics in a unique and simple way. It has an important role in image analysis and pattern recognition (Mohanaiah et al., 2013). The statistical-based texture features contain various categories such as first order, second-order statistical methods and Local Binary Pattern and so forth (Ahmadvand and Daliri, 2016). The features are extracted directly from an image that is called as first order statistics. In second order statistical texture analysis, texture information depends upon the probability of finding a pair of gray-levels at random distances and orientations over an entire image.

The paper is organised as follows. The research methodology is described in Section 2. The experimental results are presented and discussed in Section 3. Finally, a conclusion of this paper is mentioned in Section 4.

\section{Research method}

The method is classified into three main phases, which are image pre-processing, and enhancement using HTT, GLCM texture feature extraction, and image classification using support vector machine (SVM) classifier. An input MRI brain images in a collected dataset is resized into certain $\mathrm{M} \times$ $\mathrm{N}$ pixels. The proposed model of brain image classification is shown in below Figure 2. Testing and Training dataset used as an input of the system model, and then image resizing operation has applied to both datasets.

\subsection{Hierarchical transformation technique}

In phase-1, MRI brain image pre-processing and enhancement process of HTT method is helpful to enhance the image classification performance. The new HTT method has been constructed by using the combination of top-hat, bottom-hat morphological operation and some mathematical operation with an optimum size of disk-shaped mask selection.

The disk-shaped mask is more commonly used one at medical-based images compare with other masks like line, octagon, square, and pair shaped masks. The size of mask is distinct from one to another image. So that selection of optimum disk-shaped mask has achieved by lowest absolute mean brightness error (AMBE) with highest peak signal to noise ratio (PSNR) values of an image.

Figure 1 MRI brain image hierarchical transformation using morphological and mathematical operation,

(a) original MRI image (b) top-hat transformed image

(c) bottom-hat transformed image (d) enhanced image

(e) image complement

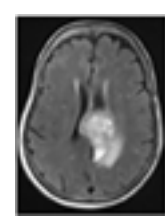

(a)

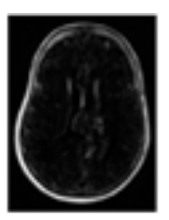

(b)

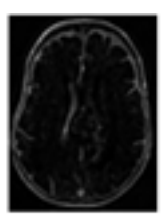

(c)

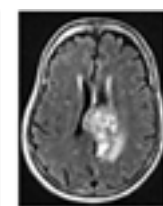

(d)

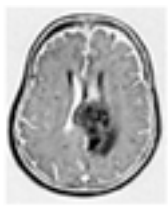

(e)
The original rescaled MRI brain image with optimum disk-shaped mask selection is represented in Figure 1(a). The top-hat transform does a morphological operation to increase the contrast between the objects and gaps that separate them from each other as shown in Figure 1(b). And bottom-hat transform is applied on original gray scale image which delivers the difference between closing and greyscale image as represented in Figure 1(c).

Then a bottom-hat image is subtracted from the sum of an original image and top-hat image which is named as enhanced image described in Figure 1(d). Finally, image complement operation has applied on obtained enhanced image depends upon the intensity valleys as shown in Figure 1(e).

\subsection{Texture feature extraction}

During this second phase, the second order GLCM statistical texture features are obtained from above resultant hierarchical transformed MRI brain image. The GLCM, introduced by Haralick is most popular second order texture statistical feature that can describe well about the spatial relationship between pixels of various gray levels (Quarat-UI-Ain, 2010; Tourrassi, 1990). It has two following levels:

1 to calculate the co-occurrence matrix

2 to compute the texture feature based on the co-occurrence matrix. 
Figure 2 Schematic diagram for proposed model of MRI brain image classification using HTT-based GLCM statistical texture feature

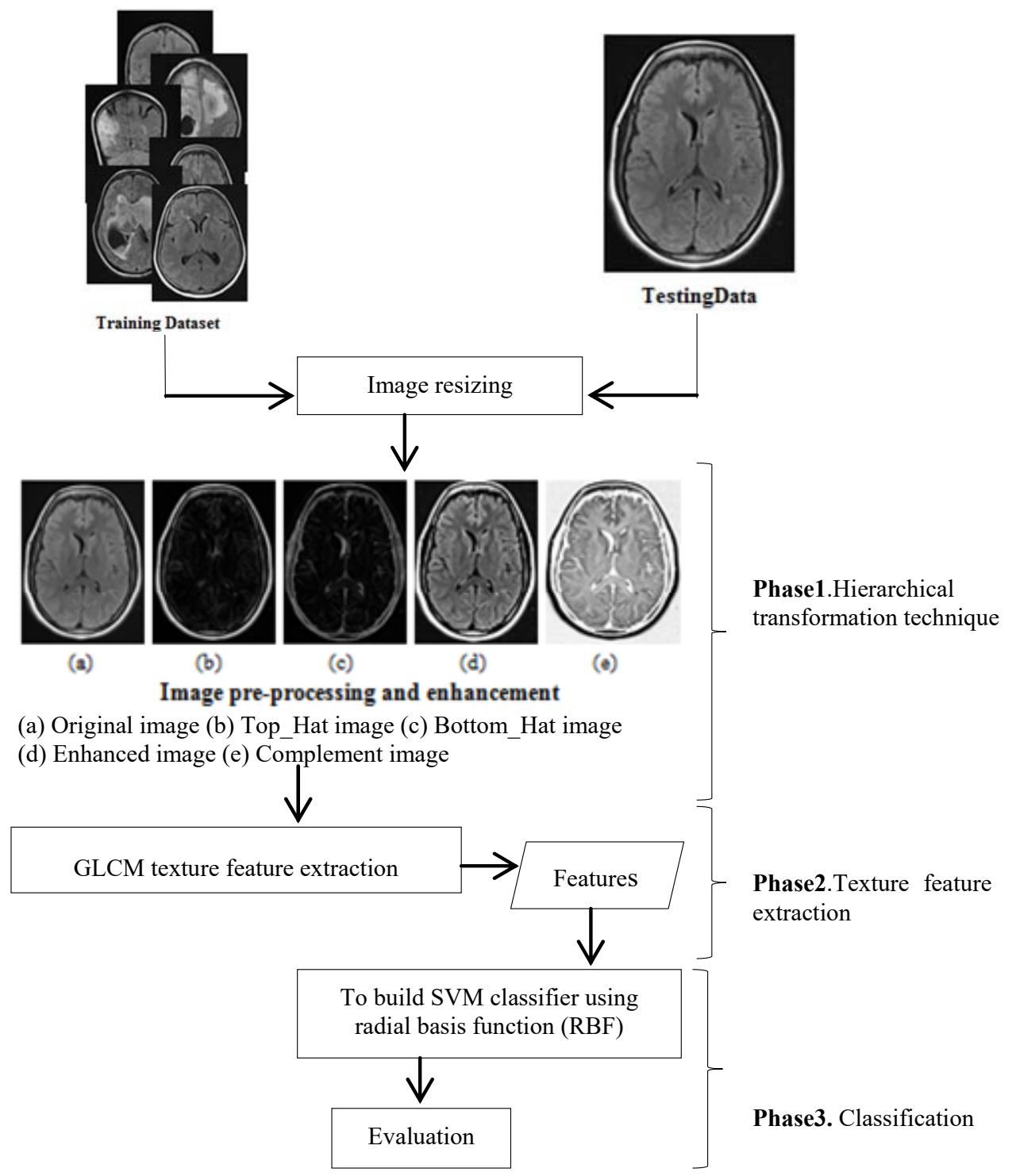

Table 1 The outcome of sample selected GLCM texture features from hierarchical transformed image

\begin{tabular}{|c|c|c|c|c|c|c|c|c|c|c|}
\hline \multirow{3}{*}{$\begin{array}{l}\text { MRI brain } \\
\text { images }\end{array}$} & \multicolumn{10}{|c|}{ GLCM texture features } \\
\hline & \multicolumn{2}{|c|}{ Contrast } & \multicolumn{2}{|c|}{ Correlation } & \multicolumn{2}{|c|}{ Energy } & \multicolumn{2}{|c|}{ Entropy } & \multicolumn{2}{|c|}{ Homogeneity } \\
\hline & Min & $\operatorname{Max}$ & Min & $\operatorname{Max}$ & Min & $\operatorname{Max}$ & Min & $\operatorname{Max}$ & Min & $\operatorname{Max}$ \\
\hline SampleImage1 & 0.1774 & 0.3259 & 0.9835 & 0.9910 & 0.3331 & 0.3368 & 1.5330 & 1.5830 & 0.9581 & 0.9668 \\
\hline SampleImage2 & 0.1787 & 0.3352 & 0.9909 & 0.9831 & 0.3331 & 0.3383 & 1.5117 & 1.5732 & 0.9567 & 0.9675 \\
\hline
\end{tabular}

GLCM is a two-dimensional histogram in which $(x, y)^{\text {th }}$ element is the event frequency $x$ that occurs with $y$. The relative distance between the pixel pair (d), angle $\theta$ (at $0^{\circ}$-horizontal, $90^{\circ}$ - vertical, $45^{\circ}$-along the positive diagonal, and $135^{\circ}$-along with negative diagonal) and gray scales $x$ and $y$, thereby, computes how often a pixel with intensity $x$, occurs in relation with another pixel $j$ at a certain distance (d) and orientation $\theta$. Most commonly used texture feature extraction is GLCM. In the classical paper (Haralick et al., 1973), Haralick proposed the fourteen textural features from the GLCM and from this only six of the textural features are judged to be the most significant that is specified in Otsu (1979).

In this procedure, calculation of GLCM was formed and contrast, correlation, entropy, homogeneity, and energy statistical texture features are obtained from above pre-processed and enhanced hierarchical transformed brain image.

The following GLCM statistics textures features are taken into consideration are: 
- Contrast: measures the intensity contrast between a pixel and it neighbour over the whole image. It can be calculated as,

$$
\sum_{x, y=0}^{N-1} P_{x y}(x-y)^{2}
$$

- Correlation: the correlation returns the degree of correlation a pixel has to its neighbour over the whole image. The range of correlation is -1 or 1 .

$$
\sum_{x, y=0}^{N-1} P_{x y} \frac{(x-\mu)(y-\mu)}{\sigma^{2}}
$$

- Energy: the computation of energy can be defined as, the sum of squared elements in the GLCM.

$$
\sum_{x, y=0}^{N-1}\left(P_{x y}\right)^{2}
$$

- Entropy: entropy is a scalar quantity which can be defined as the statistical measure of randomness. And it is helpful to identify the feature of an input image. The mathematical formulation of entropy is given by,

$$
\sum_{x, y=1}^{N-1} \log (P(i, j)) * P(i . j)
$$

- Homogeneity: it returns a value that measures the closeness of the element distribution in the gray level co-occurrence matrix (GLCM) to GLCM diagonal. Homogeneity value is 1 for a diagonal GLCM.

$$
\sum_{x, y=0}^{N-1} \frac{P_{x y}}{1+(x-y)^{2}}
$$

where $P_{x y}$ is an element $x, y$ of the normalised symmetrical GLCM. N denotes the number of gray levels in the image as specified by the number of levels in under quantisation on the GLCM. The symbol $\mu$ represents the value of GLCM mean, and it can be represented as follows,

$$
\mu=\sum_{x, y=0}^{N-1} x P_{x y}
$$

The symbol $\sigma^{2}$ indicates the variance of the intensities of all reference pixels $(x, y)$ in the relationships that contributed to the GLCM, computed as,

$$
\sigma^{2}=\sum_{x, y=0}^{N-1} P_{x y}(x-\mu)^{2}
$$

The above extracted statistical texture features are fed into SVM classifier to training and testing the performance of classifier to diagnose the MRI brain images into normal and abnormal.

\subsection{SVM classification}

An SVM classifier is a binary classification method. It is a most attractive and systematic technique for two class issues. SVM trains the above texture feature itself by using mathematical formulations which are known the kernel methods that map the training dataset into kernel space. This mapping generates patterns that will make classification work easier. There are four types of kernel functions are available in SVM such as linear, polynomial, radial basis function, and quadratic. Among this RBF kernel is helpful in vectors which are non-linearly mapped to a very high dimensional feature space. And RBF kernel increase overall accuracy compare with other kernel function has been proved (Yekkehkhany et al., 2014). So that in this proposed work, SVM classifier is used to recognise the pattern of MRI brain normality and abnormality.

\section{Experimental results}

The MRI brain image dataset in DICOM format was collected from Tirunelveli Govt. Hospital, Tamil Nadu. The pre-processing and enhancement is required to an image in order to get a better texture feature extraction and image classification. Totally, 40 normal and 40 abnormal images are taken as image dataset. Here 20 normal and 20 abnormal images have been tested. The performance evaluation of this proposed system is analysed by the statistical measures such as sensitivity, specificity, and accuracy.

The mathematical formulation of sensitivity can be defined as,

\begin{tabular}{|c|c|c|c|c|}
\hline \multirow{2}{*}{$\begin{array}{l}\text { Feature } \\
\text { extraction } \\
\text { technique }\end{array}$} & \multirow{2}{*}{ Kernel type } & \multirow{2}{*}{$\begin{array}{c}\text { Actual } \\
\text { class }\end{array}$} & \multicolumn{2}{|c|}{ Predicted class } \\
\hline & & & Normal & Abnormal \\
\hline \multirow[t]{8}{*}{ GLCM } & Polynomial & Normal & $70 \%$ & $30 \%$ \\
\hline & & Abnormal & $70 \%$ & $30 \%$ \\
\hline & Linear & Normal & $80 \%$ & $20 \%$ \\
\hline & & Abnormal & $70 \%$ & $30 \%$ \\
\hline & Quadratic & Normal & $65 \%$ & $35 \%$ \\
\hline & & Abnormal & $60 \%$ & $40 \%$ \\
\hline & Radial basis & Normal & $45 \%$ & $55 \%$ \\
\hline & & Abnormal & $50 \%$ & $50 \%$ \\
\hline
\end{tabular}

$$
\begin{aligned}
& \text { Sensitivity }=\frac{T P}{T P+F N} * 100 \\
& \text { Specificity }=\frac{T N}{T N+F P} * 100
\end{aligned}
$$

Table 2 Average performance of SVM classifier for GLCM texture feature extraction technique with different kernel functions (see online version for colours)

And accuracy is computed by the following mathematical formulation as,

$$
\text { Accuracy }=\frac{T P+T N}{T P+T N+F N+F P} * 100
$$

True positive $(T P)$ and true negative $(T N)$ represent the correctly classified positive cases and negative cases respectively. False positive $(F P)$ and false negative $(F N)$ 
represent the incorrectly classified negative cases and positive cases respectively.

The aim of this work is to recognise to MRI brain images with appropriate texture features. Here GLCM and HTT-based GLCM texture feature extraction techniques also are compared with different SVM kernel functions.

Figure 3 A statistical measurement of GLCM texture feature extraction with different kernel function (see online version for colours)

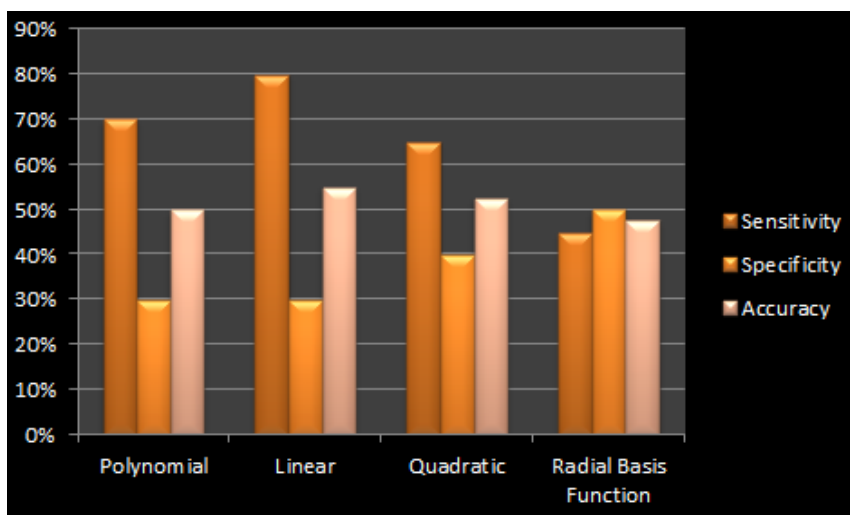

Table 3 Percentage of classification accuracy for GLCM texture feature extraction technique (see online version for colours)

\begin{tabular}{lccc}
\hline $\begin{array}{l}\text { Statistical measures/ } \\
\text { name of the kernel }\end{array}$ & Sensitivity & Specificity & Accuracy \\
\hline Polynomial & $70 \%$ & $30 \%$ & $50 \%$ \\
Linear & $80 \%$ & $30 \%$ & $55 \%$ \\
Quadratic & $65 \%$ & $40 \%$ & $52.5 \%$ \\
Radial basis function & $45 \%$ & $50 \%$ & $47.5 \%$ \\
\hline
\end{tabular}

Table 4 Average performance of SVM classifier for proposed HTT-based GLCM texture feature extraction technique with different kernel functions (see online version for colours)

\begin{tabular}{|c|c|c|c|c|}
\hline \multirow{2}{*}{$\begin{array}{l}\text { Feature } \\
\text { extraction } \\
\text { technique }\end{array}$} & \multirow{2}{*}{ Kernel type } & \multirow{2}{*}{$\begin{array}{c}\text { Actual } \\
\text { class }\end{array}$} & \multicolumn{2}{|c|}{ Predicted class } \\
\hline & & & Normal & Abnormal \\
\hline \multirow{8}{*}{$\begin{array}{l}\text { HTT-based } \\
\text { GLCM }\end{array}$} & \multirow[t]{2}{*}{ Polynomial } & Normal & $55 \%$ & $45 \%$ \\
\hline & & Abnormal & $45 \%$ & $55 \%$ \\
\hline & \multirow[t]{2}{*}{ Linear } & Normal & $65 \%$ & $35 \%$ \\
\hline & & Abnormal & $35 \%$ & $55 \%$ \\
\hline & \multirow[t]{2}{*}{ Quadratic } & Normal & $65 \%$ & $35 \%$ \\
\hline & & Abnormal & $35 \%$ & $65 \%$ \\
\hline & \multirow[t]{2}{*}{ Radial basis } & Normal & $70 \%$ & $30 \%$ \\
\hline & & Abnormal & $10 \%$ & $90 \%$ \\
\hline
\end{tabular}

Table 2 describes the average percentage of statistical measurement of GLCM texture feature extraction method. The percentage of diagonally shaded boxes represents the correctly classified brain images. The percentage of off-diagonal represents the incorrectly classified MRI brain images. And the graphical representations of statistical measurement of GLCM in various kernel functions are shown in below Figure 3.

Table 5 represents the average percentage of statistical measurement of proposed work. The percentage of diagonally shaded boxes describes the correctly classified brain images. The percentage of off-diagonal describes the incorrectly classified MRI brain images.

Table 5 Percentage of classification accuracy for HTT-based GLCM texture feature extraction technique (see online version for colours)

\begin{tabular}{lccc}
\hline $\begin{array}{l}\text { Statistical measures/ } \\
\text { name of the kernel }\end{array}$ & Sensitivity & Specificity & Accuracy \\
\hline Polynomial & $55 \%$ & $55 \%$ & $55 \%$ \\
Linear & $65 \%$ & $55 \%$ & $60 \%$ \\
Quadratic & $65 \%$ & $65 \%$ & $65 \%$ \\
Radial basis function & $70 \%$ & $90 \%$ & $80 \%$ \\
\hline
\end{tabular}

And the graphical representations of statistical measurement in various kernel functions for HTT-based GLCM are shown in below Figure 4.

Figure 4 A statistical measurement of novel texture feature extraction with different kernel function (see online version for colours)

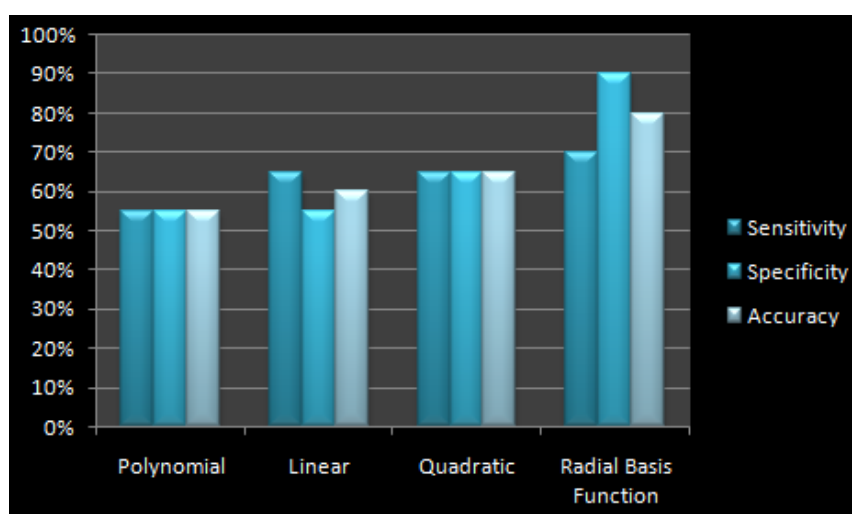

The statistical measures of sensitivity, specificity, and accuracy of GLCM in SVM with linear kernel function are compared with proposed approach in SVM radial basis kernel function.

Table 6 Comparisons of classification accuracy between conventional GLCM and proposed texture feature extraction technique (see online version for colours)

\begin{tabular}{lcc}
\hline $\begin{array}{l}\text { Statistical measures/ } \\
\text { name of the kernel }\end{array}$ & GLCM (linear) & Proposed (rbf) \\
\hline Sensitivity & $80 \%$ & $70 \%$ \\
Specificity & $30 \%$ & $90 \%$ \\
Accuracy & $55 \%$ & $80 \%$ \\
\hline
\end{tabular}

The graphical representation of above comparisons is shown in Figure 5. 
Figure 5 Comparison of statistical measures for GLCM and proposed texture feature extraction technique (see online version for colours)

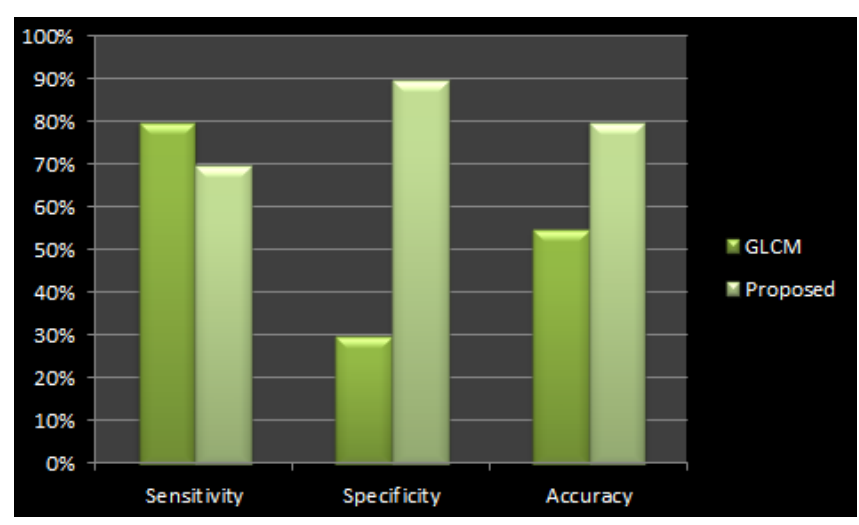

\section{Conclusions}

This paper presents an efficient method to classify the MRI brain images into a tumour and non-tumour images by using effective feature extraction technique. And the proposed work gives the promising outcomes in classification MR images. According to this experimental result, HTT-based GLCM gives $80 \%$ accuracy. The performance of this research method is also compared with various kernel functions.

\section{Acknowledgements}

The authors are thankful to Department of Radiology, Tirunelveli Government Hospital, for helping us with required image data.

\section{References}

Ahmadvand, A. and Daliri, M.R. (2016) 'A review on texture analysis methods in biomedical image processing', OMICS Journal of Radiology, April, Vol. 5, No. 2, pp.1-2.

Bandyopadhyay, S.K. (2011) 'Detection of brain tumor - a proposed method', Journal of Global Research in Computer Science, January, Vol. 2, No. 1, pp.55-63.
Castellano, G., Bonilha, L., Li, L.M. and Cendes, F. (2004) Texture Analysis of Medical Images, Neuroimage Laboratory, Faculty of Medical Sciences, State University of Campinas, Brazil.

D'cruz, N. and Sudheesh, K.V. (2015) 'Brain abnormality detection in mri image based an estimation of statistical texture measures', International Journal of Innovative science, Engineering and Technology, April, Vol. 2, No. 4, pp.633-638.

Haralick, R., Shanmugam, K. and Dinstein, I. (1973) 'Texture features for image classification', IEEE Transaction, Vol. 3, No. 6, pp.610-621.

Jing, X-J., Yu, N. and Shang, Y. (2004) 'Image filtering based on mathematical morphology and visual perception principle', Chinese Journal of Electronics, Vol. 13, pp.612-616.

Maragos, P. (1996) 'Differential morphology and image processing', IEEE Transactions on Image Processing, Vol. 5, No. 6, pp.922-937.

Materka, A. and Strzelecki, M. (1998) Texture Analysis Methods A Review, COST B11 Report, Technical University of Lodz, Poland.

Mohanaiah, P., Satyanarayana, P. and Guru Kumar, L. (2013) 'Image texture feature extraction using GLCM approach', International Journal of Scientific and Research Publication, Vol. 3, No. 5, pp.1-5.

Oritz, F. and Torres, F. (2004) 'Vectorial morphological reconstruction for brightness elimination in colour image', Real-Time Imaging, Vol. 10, No. 6, pp.379-387, Elsevier.

Otsu, N. (1979) 'A threshold selection method from gray-level histogram', IEEE Transaction on System Man Cybernetics, Vol. 9, No. 1, pp.9962-9966.

Peters, R.A. (1995) 'A new algorithm for image noise reduction using mathematical morphology', IEEE Transaction on Image Processing, Vol. 4, No. 5, pp.554-568.

Quarat-UI-Ain, G.L. (2010) 'Classification and segmentaion of brain tumor using texture analysis', Recent Advances in Artificial Intelligence, Knowledge Engineering And Data Bases, pp.147-155 (journal of magazine citation), ISBN: 978-960-474-154-0, ISSN: 1790-5109.

Tourrassi, G.D. (1990) 'Journey towards computer aided dignosis-role of image texture analysis', Radiology, Vol. 213, No. 2, pp.317-320.

Yekkehkhany, B., Safari, A., Homayouni, S. and Hasanlou, M. (2014) 'A comparison study of different kernel functions for SVM-based classification of multi-temporal polarimetry SAR data', International Achieves of Photogrammetry, Remote Sensing and Spatial Information Sciences, Vol. XL-2/W3, pp.281-285. 